\title{
A PROBLEM OF THE CALCULUS OF VARIATIONS IN WHICH THE INTEGRAND IS DISCONTINUOUS*
}

BY

\section{GILBERT AMES BIISS AND MAX MASON}

In the calculus of variations the assumption is made, in dealing with integrals of the form

$$
J=\int F\left(x, y, x^{\prime}, y^{\prime}\right) d t,
$$

that $F$ is a continuous function of its four arguments. In the following pages the properties of a minimizing curve,

$$
x=\phi(t), \quad y=\psi(t),
$$

for the integral $J$ will be investigated when the function $F$, in general continuous, is allowed to have a finite discontinuity when the point $(x, y)$ passes through a given curve.

This case would arise in the treatment by calculus of variations of the physical phenomena which take place in a medium whose properties change abruptly. For example, a ray of light follows such a path from one point, 1, to another, 2 , that the time of passage is the shortest possible. $\dagger$ If we take the points 1,2 , in the $x y$-plane and assume the medium to be such that the velocity of light is given by a function $V(x, y)$ independent of $z$, then the path of the ray is a plane curve of the form (1) which minimizes the integral

$$
\int_{1}^{2} \frac{\sqrt{x^{\prime 2}+y^{\prime 2}}}{V(x, y)} d t .
$$

If abrupt changes in $V$ occur, i. e., if refracting surfaces are present, the integrand has discontinuities of the kind above mentioned.

The problem to be treated in the following pages may be stated as follows: Among all curves which connect two given points 1 and 3 lying on opposite sides of a fixed curve $L$, and which cross $L$ but once, that one $C$ is required which minimizes the sum of the two integrals

$$
\begin{aligned}
J & =\int F\left(x, y, x^{\prime}, y^{\prime}\right) d t, \\
j & =\int f\left(x, y, x^{\prime}, y^{\prime}\right) d t,
\end{aligned}
$$

* Presented to the Society October 28, 1905. Received for publication December 18, 1905.

IIn some cases the time is a maximum, but in a sense different from the one ordinarily nnderetood in the calculus of variations. See, e. g., DRUDE, Lehrbuch der Optik, p. 11. 
where the first integral is to be taken from the fired point 1 to the curve $L$, the second from $L$ to the fixed point 3 .

The intersection of the curve $L$ with the solution $C$ will be denoted by 2 , the intersection of $L$ with any comparison curve by 2 '.

Three of the necessary conditions which the curve $C_{12}$ must satisfy in order to minimize the integral are readily derivable from the known conditions in the ordinary problem of the calculus of variations. In $\S 2$ a fourth condition corresponding to the Jacobi condition will be derived, and it will be shown in $\S 3$. that the four conditions with the usual slight alterations are also sufficient.

\section{$\$ 1$. The known necessury conditions.}

All the curves considered are supposed to lie in a closed region $R$ of the $x y$ plane. This region is divided by the curve $L$,

$$
L: \quad x=a(u), \quad y=b(u)
$$

into two closed regions which contain the points 1 and 3 respectively. The two functions $F$ and $f$ in the integrals $J$ and $j$ will be assumed to have similar properties over the entire region $R .^{*}$ These properties need be stated for only one of them. In the region $R$ the function $F\left(x, y, x^{\prime}, y^{\prime}\right)$ is of class $C^{\prime \prime \prime} \dagger$ for all values of $x^{\prime}$ and $y^{\prime}$ not both zero. $F$ has also the usual homogeneity property +

or

$$
F^{\prime}\left(x, y, \kappa x^{\prime}, \kappa y^{\prime}\right)=\kappa F\left(x, y, x^{\prime}, y^{\prime}\right) \quad(\kappa>0),
$$

which makes the integral $J$ independent of the particular parameter representation chosen for the curve (1). In order to avoid complication it will further be assumed that the problem is a regular one, i. e., that the function

$$
F_{1}^{\prime}=\frac{1}{y^{\prime 2}} F_{x^{\prime} x^{\prime}}=-\frac{1}{x^{\prime} y^{\prime}} F_{x^{\prime} y^{\prime}}=\frac{1}{x^{\prime 2}} F_{y^{\prime} y^{\prime}}
$$

is everywhere different from zero.

The functions $a(u), b(u)$ defining the curve $L$ are of class $C^{\prime}$.

The EULER equations which define the extremals for the integral $J$ have the form

$$
F_{x}^{\prime}-\frac{d}{d t} F_{x^{\prime}}=0, \quad F_{y}-\frac{d}{d t} F_{y^{\prime}}=0,
$$

* In a physical problem $F$ or $f$ will usually be defined only on one side of $L$. In order to satisfy this assumption their definitions may be extended arbitrarily over the whole of $R$.

$\dagger$ By a function of class $C^{(n)}$ is meant one which with all its derivatives of order up to and including $n$, is continuous.

$\ddagger$ For an explanation of the notions and well known theorems of the calculus of variations used in the following pages, the reader is referred to PoL\%A, Lectures on the Calculus of Variations, Chicago, 1904. 
and these are equivalent to the single equation

$$
F_{x^{\prime}}-F_{x^{\prime} y}+F_{1}^{\prime}\left(x^{\prime} y^{\prime \prime}-x^{\prime \prime} y^{\prime}\right)=0 \text {. }
$$

Equation (6) is equivalent to the system

$$
d x=\cos \Gamma, \quad d y=\sin \Gamma, \quad d \Gamma=\frac{F^{\prime}, l}{d s}-F_{s, \prime^{\prime}},
$$

where $s$ is the length of arc and $\Gamma$ the direction of the tangent on the curve. In the third equation the arguments of $F_{, \prime \prime \prime}, F_{x, y \prime^{\prime}}, F_{1}^{\prime}$, are $x, y, \cos \Gamma, \sin \Gamma$.

The assumptions made on $F^{\prime}$ and the existence theorems ${ }^{*}$ on differential equations, justify the following statement:

Through every point $\left(x_{0}, y_{10}\right)$ of the region $R$ theres passes one and but one extremal for the integral $J$ in the direction $\Gamma_{v}$. These curves extend from boundary to boundary of the region $R$. Analytically they may be represented, by the equations

$$
x=\Phi\left(s, x_{0}, y_{0}, \Gamma_{0}\right), \quad y=\Psi\left(s, x_{11}, y_{0}, \Gamma_{0}\right),
$$

where $\Phi$ and $\Psi$ satisfy the initial conditions,

$$
\begin{aligned}
x_{0} & =\Phi\left(0, x_{0}, y_{0}, \Gamma_{0}\right), & y_{0} & =\Psi\left(0, x_{10}, y_{0}, \Gamma_{0}\right), \\
\cos \gamma_{0} & =\Phi\left(0, x_{0}, y_{0}, \Gamma_{0}\right), & \sin \gamma_{0} & =\Psi_{s}\left(0, x_{01}, y_{0}, \Gamma_{0}\right) .
\end{aligned}
$$

A similar set of extremals exists for the integral, $j$, which will be denoted by

$$
x=\phi\left(s, x_{0}, y_{0}, \gamma_{0}\right), \quad y=\psi\left(s, x_{n}, y_{0}, \gamma_{0}\right) \text {. }
$$

The functions $\Phi$ and $\Psi$ have all first partial derivatives, and the particular second derivatives $\Phi_{x *}, \Phi_{s \Gamma_{0}}, \Psi_{* s}, \Psi_{s 1_{0} r_{0}}$, continuous. This property is true for any $\left(x_{0}, y_{0}\right)$ in $R, \Gamma_{0}$ any real value, and $s$ any value defining a point $(x, y)$ in $R$. The length of the interval within which $s$ may vary depends of course upon the choice of the initial values $x_{0}, y_{0}, \Gamma_{0}$.

Two of the conditions upon the curve $C_{123}$ which minimizes the sum of the integrals $J_{12}$ and $j_{23}$ are easily derived from the usual theory. The arcs $C_{12}$ and $C_{23}$ must minimize $J$ and $j$ with respect to other curves joining the point 1 with the point 2 and 2 with 3 respectively, so that

I. $C_{12}$ must be an extremul for the integral $J, i$. e., one of the set ( 7$) . C_{23}$ must be an extremal for the integral $j, i$. e., one of the set (8).

II. If $C_{12}$ and $C_{23}$ satisfy condition I, then, according to Legendre, the functions $F_{1}$ and $f_{1}$ must be positive along $C_{12}$ and $C_{23}$, in order that there may be a minimum and not a maximum.

On account of the assumption that the problem is regular the Weicrstrass

* See Bliss, Annals of Mathematics, ser. 2, vol. 6 (19G5), p. 49. 
$E$ function is never negative. The minimum, if one exists, will therefore be a strong minimum.

The third condition is a restriction upon the directions of the extremals $C_{12}$ and $C_{23}$ at their intersection with the curve $L$.

III. The directions $\Gamma, \gamma, \theta$, of the extremals $C_{12}, C_{23}$ ard the curve $L$ respectively, at their intersertion point $2\left(x_{2}, y_{2}\right)$, must satisfy the relation

$$
\begin{aligned}
& F_{x^{\prime}}\left(x_{2}, y_{2}, \cos \Gamma, \sin \Gamma\right) \cos \theta+F_{y^{\prime}}\left(x_{2}, y_{2}, \cos \Gamma, \sin \Gamma\right) \sin \theta \\
& \quad=f_{x^{\prime}}^{\prime}\left(x_{2}, y_{2}, \cos \gamma, \sin \gamma\right) \cos \theta+f_{y^{\prime}}\left(x_{2}, y_{2}, \cos \gamma, \sin \gamma\right) \sin \theta .
\end{aligned}
$$

A similar condition has been proved by BoLzA* in the problem which corresponds to reflection in the way that the problem here considered corresponds to refraction. In the case of reflection but one integral $J$ is involved. The proof in the present case is, however, so similar that it need not be given in detail. It consists in setting up a family of curves depending upon a parameter $\alpha$, the curve $C_{123}$ being given for the value $\alpha=0$. Each of the curves joins 1 and 3 and crosses $L$ once at a point $2^{\prime}$. The sum of the integrals, $J_{12^{\prime}}+j_{2^{\prime} 3}$, taken along one of these curves, is a function of $\alpha$, and its derivative must vanish for $\alpha=0$. After an integration by parts, well known in the calculus of variations, the integrals in the expression for the derivative vanish because $C_{12}$ and $C_{23}$ are extremals, and the condition (9) results.

In the problem of the refraction of a light ray at the surface between two homogeneous media the functions $F$ and $f$ are

$$
\frac{\sqrt{x^{\prime 2}+y^{\prime 2}}}{V}, \frac{\sqrt{x^{\prime 2}+y^{\prime 2}}}{v}
$$

where $V$ and $v$ are the constant velocities of light in the two media. Equation (9) becomes

$$
\frac{\cos (\Gamma-\theta)}{V}=\frac{\cos (\gamma-\theta)}{v},
$$

or

$$
\frac{\sin i}{\sin r}=\frac{v}{V},
$$

where $i$ and $r$ are the angles of incidence and refraction respectively.

\section{§2. The Jacobi condition.}

The following proof of the fourth necessary condition is geometrical and does not cover some cases in which the envelope of the set of extremals used has a singular point of a special kind. This objection holds also for the proof in

* Bolz A, loc. cit., p. 151. 
geometrical form given originally by KNESER* for the ordinary problem of the calculus of variations. The method has the advantage, however, of being relatively simple, and one who is familiar with the analytical discussion of the second variation will readily find the more comprehensive analytical proof. The proof will be based upon the properties of a particular family of curves consisting of a set of extremals for $J$ and a set for $j$. The existence of these sets is a consequence of the statement on page 327.

Suppose that $C_{123}$ is a curve joining the points 1 and 3 , intersecting $L$ at the point 2 , and satisfying the conditions I, II, III of the preceding section. Let $C_{12^{\prime}}$ be a neighboring extremal to $C_{12}$ through the point 1 intersecting the curve $L$ at a point 2 ' (see figure 1). It will be shown that by equation (9) the direction of $C_{1{ }^{\prime}}^{\prime}$ at $2^{\prime}$ defines uniquely another direction at that point, through which a new extremal for the integral $j$ may be passed. The one parameter set of extremals for $J$ through the point 1 defines in this way another one-parameter set for $j$

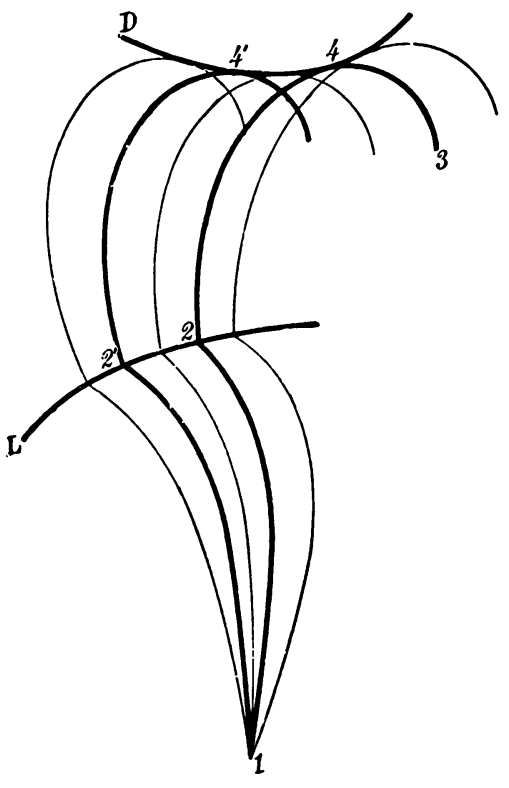

Fia. 1. having initial points on the curve $L$. It is to be proved that the $\operatorname{arc} C_{12^{3}}^{\gamma}$ cannot minimize the sum of the two integrals if it contains a point of contact with an envelope of this second set.

Consider the extremals

$$
C_{12^{\prime}}: \quad x=\Phi\left(s, x_{1}, y_{1}, \Gamma\right), \quad y=\Psi\left(s, x_{1}, y_{1}, \Gamma\right)
$$

with parameter $\Gamma$, formed from equations $(7)$ by giving $x_{0}, y_{0}$ the values of the coïrdinates of the point 1 . For the present it will be assumed that the point 2 is not conjugate to the point 1 , so that the determinant

$$
\Delta=\left|\begin{array}{ll}
\Phi_{*} & \Phi_{\Gamma} \\
\Psi_{s} & \Psi_{\Gamma}
\end{array}\right|
$$

is different from zero for the values of $s$ and $\Gamma$ defining the point 2.

It will be convenient to use the value of $u$, the parameter of the curve $L$, at the point $2^{\prime}$ as the parameter of the family instead of $\Gamma$. The change may be effected by solving the equations

$$
\Phi\left(s, x_{1}, y_{1}, \Gamma\right)=a(u), \quad \Psi\left(s, x_{1}, y_{1}, \Gamma\right)=b(u)
$$

* Mathematische Annalen, vol. 50 (1898), p. 27. Compare with his Lelhbruch der Variationsrechnung (Braunschweig, 1900), p. 93. 
for $s$ and $\Gamma$ in terms of $u$ and substituting the value of $\Gamma$ in the equations (10). The usual theorems on implicit functions show that $s$ and $\Gamma$ are defined as functions of $u$ of class $C^{\prime}$, since by the hypothesis on the extremal $C_{12}$ the above equations have a set of solutions corresponding to the point 2, and the determinant $\Delta$ is different from zero at 2. After substituting the value of $\Gamma$, the equations (10) take the form*

$$
C_{12}: \quad x=\Phi(s, u), \quad y=\Psi(s, u) .
$$

In the equation (9) $x_{2}, y_{2}, \theta$ may be expressed by the functions of $u$ which define the curve $L$, and $\Gamma$ by the function of $u$ derived from (11) which defines the directions of the extremals $C_{1 z^{\prime}}$ at the points $2^{\prime}$. The equation (9) formed in this way for the point $2^{\prime}$ is then one between $u$ and $\gamma$ which by hypothesis has one solution corresponding to the point 2. Furthermore the derivative for $\gamma$ is easily found with the help of equations (4) to be

$$
f_{1} \sin (\theta-\gamma),
$$

which is different from zero at the point 2, unless $C_{23}$ is tangent to $L$. Therefore $\gamma$ is defined as a function of $u$ of class $C^{\prime}$, unless $C_{23}$ is tangent to $L$, and the set of extremals for $j$ with initial points on $L$ is given by the equations

$$
x=\phi(s, u), \quad y=\psi(s, u),
$$

formed by substituting $a(u), b(u), \gamma(u)$ for $x_{0}, y_{0}, \gamma$ in equations (8).

in case $C_{23}$ is tangent to $L$ we could reverse the procedure, starting from a set of extremals for $j$ through 2 , and determining a set for $\dot{J}$ with initial points on $L$, by means of equation (9). Therefore, unless both $C_{12}$ and $C_{23}$ are tangent to $L$ at 2 , it may be assumed without restriction that $C_{23}$ is the one not tangent, and the above development holds. It will be assumed that this singular case does not occur for the problem under consideration, $i$. e., that $C_{12}$ and $C_{23}$ are not both tangent to $L$.

The results obtained may be restated in the following theorem :

If $C_{123}$ is a curve satisfying the conditions I, II, III, then to each extremal $C_{12}$ of the integral $J$ near $C_{12}$ there corresponds an extremal of the integral $j$ which, with $C_{12^{\prime}}$, satisfies the corner condition III at the point 2'. The oneparameter set of extremals of $J$ through the point 1 , defines in this way another one-parameter set belonging to $j$ with initial points on the curve $L$. The equations of the two sets may be put in the forms (11) and (12) with u as parameter.

Suppose that the extremals (12) have an envelope $D$ which touches the extremal $C_{2^{\prime} 4^{\prime}}$ at the point $4^{\prime}$, and the extremal $C_{23}$ at 4 , and suppose that the direction $2^{\prime} 4^{\prime}$ on $C_{2^{\prime} 4^{\prime}}$ coincides with $4^{\prime} 4$ on $D \dagger$ (see figure 1 ).

\footnotetext{
* For convenience the same symbols $\Phi$ and $\Psi$ are used for the equations in this new form.

$\dagger$ The envelope $D$ will always bave a branoh $4^{\prime} 4$ coinciding in direotion with $2^{\prime} 4^{\prime}$ unless it has a cusp of special kind at the point 4 . See p. 332.
} 
The integral $J$ taken along $C_{12^{\prime}}$, plus the integral $j$ taken along $C_{2^{\prime} 4^{\prime}}$ and $D_{4^{\prime} 4}$, apparently depends upon the choice of the extremal $C_{12^{\prime}}$, that is, upon the parameter $u$. But in reality this sum is a constant, as may be shown by forming its derivative with respect to $u$.

The derivative of $J_{12}$, is

$$
\begin{aligned}
d J_{12^{\prime}} & =\frac{d}{d u} \int_{1}^{a^{\prime}} F\left(\Phi, \Psi, \Phi^{\prime}, \Psi^{\prime}\right) d s \\
& =\left.F^{\prime} \frac{d s}{d u}\right|^{2 \prime}+\int_{1}^{2^{\prime}}\left\{F_{x} \Phi_{u}+F_{y} \Psi_{u}+F_{x^{\prime}} \Phi_{u}^{\prime}+F_{y^{\prime}} \Psi_{u}^{\prime}\right\} d s \\
& =F_{d u}^{d s}+F_{x^{\prime}} \Phi_{u}+\left.F_{y^{\prime}} \Psi_{u}\right|^{2 \prime}+\int_{1}^{\prime^{\prime}}\left\{\left(F_{x}-F_{{ }^{\prime}}^{\prime}\right) \Phi_{u}+\left(F_{y}-F_{y^{\prime}}^{\prime}\right) \Psi_{u}\right\} d s
\end{aligned}
$$

since $\Phi_{u}$ and $\Psi_{u}$ vanish for $s=0$. This follows because all the extremals (11) pass through the point 1 for $s=0$, i. e.,

$$
x_{1}=\Phi(0, u), \quad y_{1}=\Psi(0, u) .
$$

The integral in the above equation vanishes on account of (5) which is satisfied by the extremals (11). The remainder of the right member may be transformed by equation (3), so that

$$
\frac{d J_{1 \underline{x}^{\prime}}}{d u}=\left(\Phi^{\prime} \frac{d s}{d u}+\Phi_{u}\right) F_{x^{\prime}}+\left.\left(\Psi^{\prime} \frac{d s}{d u}+\Psi_{u}\right) F_{y^{\prime}}\right|^{2^{\prime}} .
$$

Now the value of $s$ corresponding to the point $2^{\prime}$ is the solution of the equations

and hence

$$
\Phi(s, u)=a(u), \quad \Psi(s, u)=b(u),
$$

$$
\frac{d J_{11^{\prime}}}{d u}=F_{x^{\prime}}\left(\Phi, \Psi, \Phi^{\prime}, \Psi^{\prime}\right) \frac{d a}{d u}+\left.F_{y^{\prime}}\left(\Phi, \Psi, \Phi^{\prime}, \Psi^{\prime}\right) \frac{d b}{d u}\right|^{2^{\prime}} .
$$

In a similar manner it is found that

$$
\begin{aligned}
& \frac{d j_{2^{\prime} \psi^{\prime}}}{d u}=\frac{d}{d \bar{u}} \int_{2^{\prime}}^{4^{\prime}} f\left(\phi, \psi, \phi^{\prime}, \psi^{\prime}\right) d s
\end{aligned}
$$

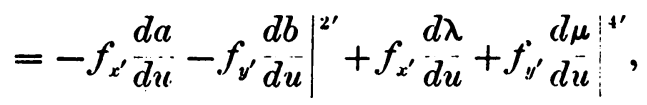

where $\lambda(u), \mu(u)$ are the functions defining $x$ and $y$ for the envelope $D$. In deriving this result it should be remembered that the extremals (12) intersect $L$ for the parameter value $s=0$, a fact expressed by the equations

$$
\phi(0, u)=a(u), \quad \psi(0, u)=b(u) ;
$$


and furthermore that $\lambda$ and $\mu$ are defined by the equations

$$
\lambda(u)=\phi(s, u), \quad \mu(u)=\psi(s, u),
$$

in which $s$ is the solution of

in terms of $u$.

$$
\delta=\left|\begin{array}{ll}
\phi_{.} & \phi_{u} \\
\psi_{,} & \psi_{u}
\end{array}\right|=0
$$

If the envelope $D$ has not a singular point at 4, the expression (14) may be put into a more convenient form. Since

$$
\frac{d \lambda}{d u}=\phi_{s} \frac{d s}{d u}+\phi_{u}, \quad \frac{d \mu}{d u}=\psi_{s} \frac{d s}{d u}+\Psi_{u},
$$

where $s$ is the solution of $\delta=0$, it follows readily that

$$
\left|\begin{array}{ll}
\text { क. } & \frac{d \lambda}{\overline{d u}} \\
\psi . & \frac{d \mu}{\bar{d} u}
\end{array}\right|=0 .
$$

Since the extremal is assumed to be free of singular points $\phi_{\text {c and }} \psi$, cannot vanish together. Unless $D$ has a singular point at 4 the functions $d \lambda / d u$, $d \mu / d u$ do not vanish together in the neighborhood of the point 4 , and there exists a function $\kappa$ of constant sign such that

$$
\phi_{c}=\kappa \frac{d \lambda}{d u}, \quad \psi_{c}=\kappa \frac{d \mu}{d u} .
$$

It may be assumed that $\kappa>0$, for changing $u$ to $-u$ changes the sign of $\kappa$. Substituting these values of $\phi_{c}$ and $\psi_{c}$ in the last two terms of (14) and applying (2) and (3) there results :

$$
\begin{aligned}
\frac{d j_{z^{\prime} \psi^{\prime}}}{d u}=-f_{x^{\prime}}\left(\phi, \psi, \phi^{\prime}, \psi^{\prime}\right) \frac{d a}{d u} & \\
& -\left.f_{y^{\prime}}\left(\phi, \psi, \phi^{\prime}, \psi^{\prime}\right) \frac{d b}{d u}\right|^{2^{\prime \prime}}+\left.f\left(\lambda, \mu, \lambda^{\prime}, \mu^{\prime}\right)\right|^{\iota^{\prime}} .
\end{aligned}
$$

In case the envelope $D$ has a singular point at $4^{\prime}$ the above transformation of equation (14) may not be possible, and the proof given here may fail.

Finally

$$
\begin{aligned}
\frac{d j_{4^{\prime}}}{d u} & =\frac{d}{d u} \int_{\mathbf{t}^{\prime}}^{4} f\left(\lambda, \mu, \lambda^{\prime}, \mu^{\prime}\right) d u \\
& =-\left.f\left(\lambda, \mu, \lambda^{\prime}, \mu^{\prime}\right)\right|^{4^{\prime}} .
\end{aligned}
$$

The sum of the three expressions (13), (15) and (16) vanishes identically on 
account of equation (9). The theorem corresponding to KNESER's theorem in the ordinary theory follows at once :

If the envelope $D$ of the extremals $C_{x^{\prime} t^{\prime}}$ has not a singular point at its point of contact with $C_{23}$, then the value of the sum of the integrals $J_{12}$ taken along the extremal $C_{12^{\prime}}$ firom the point 1 to the curve $L, j_{2^{\prime} 4^{\prime}}$ taken along the extremal $C_{2^{\prime} t^{\prime}}$ from the curve $L$ to the point of contact of $C_{2^{\prime} \prime^{\prime}}$ with the envelope $D$, and $j_{t^{\prime} \mid}$ taken along $D$ from the point of contact with $C_{2^{\prime} t^{\prime}}$ to the point of contact with $C_{24}$, always has the same value as the sum of $J_{12}$ and $j_{24}$ taken along the extremals $C_{12}$ and $C_{24}$ respectively (see figure 1 ).

With this theorem in mind it is easy to see that the extremal $C_{123}$ cannot minimize $J_{12}+j_{23}$ if the point 4 lies on the arc $C_{23}$ and is not a singular point of the envelope $D$. For $D$ cannot be an extremal, since under the assumptions there exists but one extremal through a given point in a given direction, and therefore the arc of $D$ between $4^{\prime}$ and 4 could be replaced by another curve giving a smaller value to $j$. It follows that 1 and 3 could be joined by a curve through a point $2^{\prime}$ of $L$, giving $J_{1 y^{\prime}}+j_{2^{\prime} 3}$ a smaller value than

$$
J\left(C_{1 z^{\prime}}\right)+j\left(C_{2^{\prime 4^{\prime}}}\right)+j\left(D_{1^{\prime} 4}\right)+j\left(C_{2^{\prime} 3}\right),
$$

and hence smaller than $J\left(C_{12}\right)+j\left(C_{23}\right)$.

The particular cases where 1 and 2 , or 2 and 3 are conjugate points on $C_{12}$ or $C_{23}$ need special discussion. If 2 and 3 are conjugate the point 4 lies between them. For the determinant $\delta$ is a solution of JACOBI's differential equation, a linear homogeneous differential equation of second order, having in this case, since 2 and 3 are conjugate, a solution which vanishes at 2 and 3. By the well known S'rURM's theorems concerning the separation of the roots of such solutions, $\delta$ will have a zero between 2 and 3 , and $C_{123}$ will not minimize the integral. If 1 and 2 were conjugate in $C_{12}$, but 2 and 3 not conjugate in $C_{23}$, the start should be made with the one-parameter set of extremals through 3 instead of through 1 , and the same conclusion would be reached $-C_{123}$ is not a minimizing curve. In case both 1, 2 and 2, 3 were conjugate pairs, a point $1^{\prime}$ on $C_{12}$ between 1 and 2 could be used as a starting point instead of 1 , and it would follow that the contact point 4 would again be between 2 and 3 , since they are conjugate, and $C_{123}$ would not minimize $J+j$. To summarize :

IV. The extremal arcs $C_{12}$ and $C_{23}$, including the end points, can contain no points conjugate to their initial points 1 and 2 , and the envelope of the extremals $C_{2^{\prime} \mathbf{4}^{\prime}}$ must not touch the arc $C_{23}$ between 2 and 3.*

* The proof given above shows that the envelope cannot touch $C_{23}$, even at the point 3 , unless there is a singular point at 3 . On the other hand the proof fails in case the envelope touches between 2 and 3 and has a singular point. The condition in this case may, however, be derived analytically by consideration of the second variation. 


\section{§3. The sufficient conditions.}

Suppose a curve $C_{1: 3}$ exists which crosses $L$ but once (at 2) without having both arcs $C_{12}$ and $C_{23}$ tangent to $L$, and satisfies the conditions I, II, III, of the preceding sections besides the condition

IV . The extremal arcs $C_{12}$ and $C_{23}$, including the end points, contain no points conjugate to their initial points 1 and 2 , and the envelope of the extremals $C_{2^{\prime} t^{\prime}}$ does not touch the arc $C_{23}$ between 2 and 3 OR AT 3.

It will be proved by means of HILBERT's invariant integral that the curve $C_{123}$ minimizes the sum of the integrals $J+j$.

As has been shown above, the set of extremals $C_{12}$, through 1 determines a set $C_{2^{\prime} 4^{\prime}}$ for the integral $j$ which, with $C_{12^{\prime}}$, satisfy the corner condition III. Since by condition $I V^{\prime}$ the determinants $\Delta$ and $\delta$ for these two sets are different from zero between 1 and 2 and at 2 , and between 2 and 3 and at 3 respectively, it follows that the extremals

$$
x=\Phi(s, u), \quad y=\Psi(s, u),
$$

for the integral $J$, and the extremals

$$
x=\phi(s, u), \quad y=\psi(s, u),
$$

for the integral $j$, form two fields, $B$ and $b$, about the arcs $C_{12}$ and $C_{23}$ respectively. In $B$ and $b$ the derivatives $\Phi^{\prime}, \Psi^{\prime} ; \phi^{\prime}, \psi^{\prime}$, which determine the directions of the extremals, are functions of $x$ and $y$.

Now the integral

$$
J^{*}=\int\left\{F_{x^{\prime}}\left(x, y, \Phi^{\prime}, \Psi^{\prime}\right) x^{\prime}+F_{y^{\prime}}\left(x, y, \Phi^{\prime}, \Psi^{\prime}\right) y^{\prime}\right\} d t,
$$

where $\Phi^{\prime}, \Psi^{\prime}$ are these functions of $x$ and $y$, taken along any path

$$
x=x(t), \quad y=y(t),
$$

which lies in the field $B$, is independent of the path and depends only on the end points.* Furthermore it follows from the homogeneity of $F$ that $J^{*}$ taken along an extremal is equal to $J$. Similarly the integral

$$
j^{*}=\int\left\{f_{x^{\prime}}\left(x, y, \phi^{\prime}, \psi^{\prime}\right) x^{\prime}+f_{y^{\prime}}\left(x, y, \phi^{\prime}, \psi^{\prime}\right) y^{\prime}\right\} d t
$$

is independent of the path in $b$ and reduces to $j$ when the path is an extremal for the integral $j$.

Consider any curve $C^{\prime}$,

$$
C: \quad x=x(t), \quad y=y(t),
$$

* Bolza, loc. cit., p. 195. 
which crosses $L$ once at the point 6 , lies entirely in the two fields $B$ and $b$, and joins two points in $B$ and $b$ respectively. The sum $J^{*}\left(C_{56}\right)+j^{*}\left(C_{67}\right)$ is independent of the path and depends only on the end points 5 and 7. In fact, let $C^{\prime}$ be any other curve joining 5 and 7 , and crossing $L$ at $6^{\prime}$ (see figure 2 );

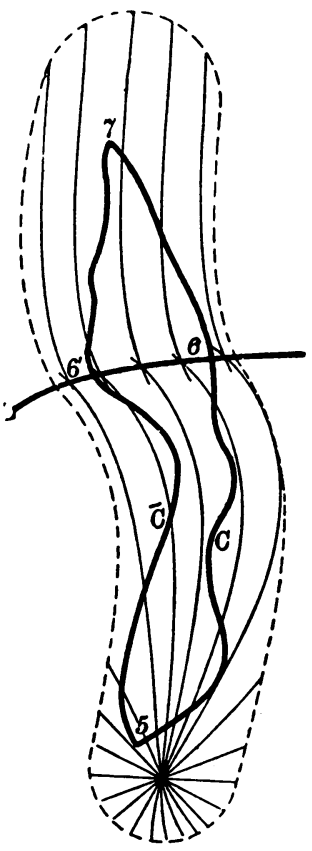

Fra. 2.

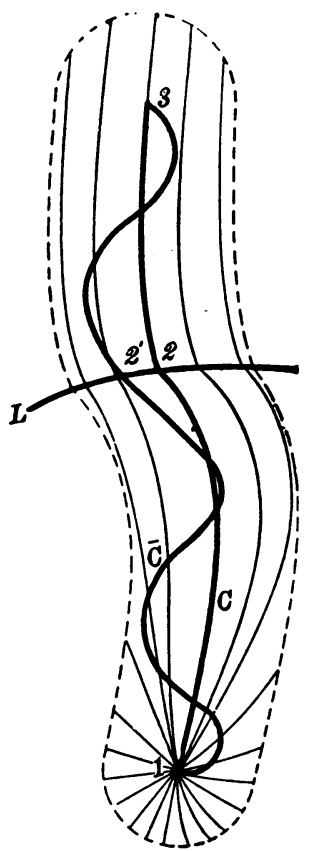

Fia. 3.

then, since the integrals $J^{*}$ and $j^{*}$ are invariant in the fields $B$ and $b$ respectively,

Therefore,

$$
\begin{aligned}
J^{*}\left(C_{56^{\prime}}\right)+J^{*}\left(L_{6^{\prime} 6}\right) & =J^{*}\left(C_{56}\right), \\
j^{*}\left(L_{66^{\prime}}\right)+j^{*}\left(C_{6^{\prime} 7}\right) & =j^{*}\left(C_{67}\right) .
\end{aligned}
$$

$$
\begin{aligned}
J^{*}\left(C_{56^{\prime}}\right)+j^{*}\left(C_{6^{\prime} 7}\right)-\left[J^{*}\left(C_{56}\right)+j^{*}\left(C_{67}\right)\right] & =-J^{*}\left(L_{66^{\prime}}\right)-j^{*}\left(L_{66^{\prime}}\right) \\
& =J^{*}\left(L_{66^{\prime}}\right)-j^{*}\left(L_{66^{\prime}}\right),
\end{aligned}
$$

since the integrand of $J^{*}$ changes sign when the direction of integration is reversed. Hence

$$
\begin{aligned}
J^{*}\left(\bar{C}_{56^{\prime}}\right)+j^{*}\left(\bar{C}_{6^{\prime}}\right)-\left[J^{*}\left(C_{56}\right)+j^{*}\left(C_{67}\right)\right] \\
=\int_{6}^{b^{\prime}}\left\{F_{x^{\prime}}\left(a, b, \Phi^{\prime}, \Psi^{\prime}\right) \frac{d a}{d u}+F_{y^{\prime}}\left(a, b, \Phi^{\prime}, \Psi^{\prime}\right) \frac{d b}{d u}\right. \\
\left.\quad-f_{x^{\prime}}\left(a, b, \phi^{\prime}, \psi^{\prime}\right) \frac{d a}{d u}-f_{v^{\prime}}\left(a, b, \phi^{\prime}, \psi^{\prime}\right) \frac{d b}{d u}\right\} d u .
\end{aligned}
$$


Since the extremals (17), (18) satisfy equation (9), the integrand of the right member is identically zero and $J^{*}+j^{*}$ is independent of the path. Furthermore, if the curve $C_{567}$ coincides with an extremal for $J$ from 5 to 6 , and with an extremal for $j$ from 6 to 7 , then

$$
J^{*}\left(C_{56}\right)+j^{*}\left(C_{67}\right)=J\left(C_{56}\right)+j\left(C_{67}\right) .
$$

Let $\bar{C}$ (figure 3 ) be any curve joining the points 1 and 2 , lying entirely in the two fields $B$ and $b$, and crossing $L$ at $2^{\prime}$, and let $C$ be the curve satisfying conditions I, II, III, IV'. Then

$$
\begin{aligned}
J^{*}\left(\bar{C}_{12^{\prime}}\right)+j^{*}\left(\bar{C}_{2^{\prime} 3}\right) & =J^{*}\left(C_{12}\right)+j\left(C_{23}\right) \\
& =J\left(C_{12}\right)+j\left(C_{23}\right) .
\end{aligned}
$$

Therefore, by substituting this value of $J\left(C_{12}\right)+j\left(C_{23}\right)$,

$$
\begin{aligned}
J\left(C_{12^{\prime}}\right)+j\left(C_{z^{\prime} 3}\right)-\left[J\left(C_{12}\right)+j\left(C_{23}\right)\right] & =J\left(\bar{C}_{12^{\prime}}\right)-J^{*}\left(\bar{C}_{12^{\prime}}\right)+j\left(\bar{C}_{2^{\prime} 3}\right)-j^{*}\left(C_{2^{\prime} 3}\right) \\
& =\int_{\bar{c}_{12^{\prime}}} E d t+\int_{\bar{C}_{23^{\prime}}} e d t,
\end{aligned}
$$

where $E$ and $e$ are the WeIErstrass $E$ functions for the fields $B$ and $b$ respectively. But as was stated in $\S 1$ the $E$ functions are nowhere negative, and they vanish only when the direction of $\bar{C}$ at any point coincides with the direction of the extremal at that point. Therefore

$$
J\left(\bar{C}_{12^{\prime}}\right)+j\left(\bar{C}_{2^{\prime} 3}\right)>J\left(C_{12}\right)+j\left(C_{23}\right),
$$

and the curve $C_{123}$ minimizes the sum of the integrals. The result may be stated in the following theorem :

$A$ curve $C_{123}$ which joins the fixed points 1,3 , and crosses the curve $L$ once at the point 2 without having both branches tangent to $L$ at 2 , minimizes the sum of the integrals .J and $j$ if it satisfies the conditions $I, I I, I I I, I V^{\prime}$.

The above discussion may be readily extended to cover the case of any number of curves of discontinuity for the integrand of the calculus of variations problem, i. e., in the case of light, to any number of refracting surfaces. The methods used above apply also with but slight modifications to the problem of the calculus of variations corresponding to the reflection of light. 